\title{
Cosmological parameters from velocities, cosmic microwave background and supernovae
}

\author{
S. L. Bridle, ${ }^{1 \star}$ I. Zehavi, ${ }^{2}$ A. Dekel, ${ }^{3}$ O. Lahav, ${ }^{3,4}$ M. P. Hobson ${ }^{1}$ and A. N. Lasenby ${ }^{1}$ \\ ${ }^{1}$ Astrophysics Group, Cavendish Laboratory, Madingley Road, Cambridge CB3 OHE \\ ${ }^{2}$ NASA/Fermilab Astrophysics Group, Fermi national Accelerator Laboratory, Box 500, Batavia, IL 60510-0500, USA \\ ${ }^{3}$ Racah Institute of Physics, The Hebrew University, Jerusalem 91904, Israel \\ ${ }^{4}$ Institute of Astronomy, Madingley Road, Cambridge CB3 OHA
}

Accepted 2000 September 5. Received 2000 August 21; in original form 2000 June 1

\begin{abstract}
A B S T R A C T
We compare and combine likelihood functions of the cosmological parameters $\Omega_{\mathrm{m}}, h$ and $\sigma_{8}$, from peculiar velocities, cosmic microwave background (CMB) and type Ia supernovae. These three data sets directly probe the mass in the Universe, without the need to relate the galaxy distribution to the underlying mass via a 'biasing' relation. We include the recent results from the CMB experiments BOOMERANG and MAXIMA-1. Our analysis assumes a flat $\Lambda$ cold dark matter $(\Lambda \mathrm{CDM})$ cosmology with a scale-invariant adiabatic initial power spectrum and baryonic fraction as inferred from big-bang nucleosynthesis. We find that all three data sets agree well, overlapping significantly at the $2 \sigma$ level. This therefore justifies a joint analysis, in which we find a joint best-fitting point and 95 per cent confidence limits of $\Omega_{\mathrm{m}}=0.28(0.17,0.39), h=0.74(0.64,0.86)$ and $\sigma_{8}=1.17(0.98,1.37)$. In terms of the natural parameter combinations for these data $\sigma_{8} \Omega_{\mathrm{m}}^{0.6}=0.54(0.40,0.73), \Omega_{\mathrm{m}} h=0.21$ $(0.16,0.27)$. Also for the best-fitting point, $Q_{\mathrm{rms}-\mathrm{ps}}=19.7 \mu \mathrm{K}$ and the age of the Universe is $13.2 \mathrm{Gyr}$.
\end{abstract}

Key words: methods: statistical - cosmic microwave background - cosmological parameters - cosmology: observations - cosmology: theory - large-scale structure of Universe.

\section{INTRODUCTION}

A simultaneous analysis of the constraints placed on the cosmological parameters by various different kinds of data is essential because each different probe typically constrains a different combination of the parameters. By considering these constraints together, one can overcome any intrinsic degeneracies to estimate each fundamental parameter and its corresponding random uncertainty. The comparison of constraints can also provide a test for the validity of the assumed cosmological model or, alternatively, a revised evaluation of the systematic errors in one or all of the data sets. Recent papers that combine information from several data sets simultaneously include Bond \& Jaffe (1998), Gawiser \& Silk (1998), Lineweaver (1998), Bahcall et al. (1999), Bridle et al. (1999), and Lange et al. (2000).

Galaxy motions relative to the Hubble flow arise from the gravitational forces caused by mass-density fluctuations; they therefore reflect the underlying distribution of matter (both dark and luminous), and can thus provide constraints on the cosmological density parameter $\Omega_{\mathrm{m}}$ and the fluctuation amplitude parameter $\sigma_{8}$. For example, constraints on the cosmological

\footnotetext{
^E-mail: s.bridle@mrao.cam.ac.uk
}

parameters were obtained by Zaroubi et al. (1997) and Freudling et al. (1999) from a likelihood analysis of the Mark III and SFI catalogues of peculiar velocities, within the framework of Cosmic Background Explorer (COBE) normalized cold dark matter (CDM) models and Gaussian fluctuations and errors. The anisotropies in the cosmic microwave background (CMB) depend on the state of the universe at the epoch of recombination, on the global geometry of space-time and on any re-ionization. Thus they provide a powerful and potentially accurate probe of the cosmological parameters (see Hu, Sugiyama \& Silk 1997 for a review). With the recent release of results from a new generation of CMB experiments BOOMERANG and MAXIMA- 1 have come a number of parameter estimation analyses, including those by Balbi et al. (2000), Lange et al. (2000) and Tegmark \& Zaldarriaga (2000). The distances of type Ia supernovae (SN) can now be measured at large redshift. Thus they can provide constraints on the acceleration of the universal expansion, and the corresponding parameters $\Omega_{\mathrm{m}}$ and $\Omega_{\Lambda}$, via a classical cosmological test based on the luminosity-redshift relation. These three cosmic probes allow direct dynamical constraints free of assumptions regarding the 'biasing' relation between the distribution of galaxies and the underlying matter density, which are unavoidable when interpreting galaxy redshift surveys.

Various analyses have been performed in which pairs of these 
data sets are used to place constraints in the $\Omega_{\mathrm{m}}, \Omega_{\Lambda}$ plane: Efstathiou et al. (1999) investigate CMB and SN; Zehavi \& Dekel (1999) explore peculiar velocities with SN. In this work, we perform a joint analysis of all three data sets. We restrict the analysis to the scale-invariant flat $\Lambda \mathrm{CDM}$ model, which is motivated by theoretical arguments based on the inflation scenario, and is consistent with CMB observations (e.g. Bond \& Jaffe 1998 find $n \sim 1$ from the COBE data; recent analyses of the BOOMERANG and MAXIMA- 1 data find $\Omega_{\mathrm{m}}+\Omega_{\Lambda} \sim 1$ ). In addition we use the nucleosynthesis constraint of $\Omega_{\mathrm{b}} h^{2} \sim 0.019$ (Burles et al. 1999; Tytler et al. 2000), although we discuss the validity of this assumption in the light of the recent measurements of the CMB second peak height.

An earlier paper (Bridle et al. 1999) investigated the combination of constraints from CMB data, the abundance of clusters of galaxies (Eke et al. 1998) and the IRAS 1.2-Jy redshift survey (Fisher, Scharf \& Lahav 1994). These data sets were found to be in good agreement, once the densities of galaxies and mass are assumed to be related via a linear biasing parameter. In this paper we focus on the implications of combining three dynamical data sets that are free of galaxy-biasing uncertainties. In Section 2 we introduce each of the data sets and outline the theory used to link the constraints from each and in Section 3 we compare and combine the constraints from the different data.

\section{THE THREE PROBES OF MASS}

\subsection{Peculiar velocities}

We consider two catalogues of galaxy peculiar velocities (PV). One, Mark III (Willick et al. 1997), contains $\sim 3000$ galaxies within $\sim 70 h^{-1} \mathrm{Mpc}\left[h \equiv H_{0} /\left(100 \mathrm{~km} \mathrm{~s}^{-1} \mathrm{Mpc}^{-1}\right)\right]$. The other, SFI (Haynes et al. 1999a,b), consists of $\sim 1300$ spiral galaxies but with a more uniform spatial coverage in a similar volume. The error per galaxy is 15-21 per cent of its distance. The constraints obtained from the two data sets were found to be very similar (e.g Zehavi \& Dekel 2000). Note that in fact, after a non-linear correction (see later) Mark III gives a slightly lower amplitude power spectrum than SFI, and a lower value of $\Omega_{\mathrm{m}}$, and therefore tends to agree even better with other constraints. We choose to perform our analysis here on the SFI catalogue.

The analysis follows in general the maximum-likelihood method of Zaroubi et al. (1997) and Freudling et al. (1999). The density and velocity fluctuations are assumed to be a random realization of a Gaussian field and to obey the linear approximation to gravitational instability (with one caveat discussed below). The likelihood of a given set of values for the cosmological parameters of interest is calculated by comparing the observed velocity correlations with those predicted by theory based on this set of parameters, under the assumption that the errors in the observed velocities are Gaussian.

The CDM power spectrum form used in the likelihood analysis has a shape parameter $\Gamma$, as provided by Sugiyama (1995, equation [3.9]), which determines the wavenumber at the peak of the power spectrum $P(k)$ in terms of $h, \Omega_{\mathrm{m}}$ and the baryonic content of the universe $\Omega_{\mathrm{b}}$. This is independent of $\Omega_{\Lambda}$, and we assume that the power spectrum is initially scale-invariant $(n=1)$. For the baryonic content we adopt the value favoured by deuterium abundance in the context of big-bang nucleosynthesis (Burles et al. 1999; Tytler et al. 2000) $\Omega_{\mathrm{b}} h^{2}=0.019$. Note that while Freudling et al. (1999) used $C O B E$-normalized models, we perform our current analysis of peculiar velocities with the amplitude of fluctuations as a free parameter. The COBE constraint enters the joint analysis later as part of the independent CMB data set. In this paper we thus choose as our free fundamental parameters the dimensionless Hubble constant $h$, the total matter density, $\Omega_{\mathrm{m}}\left(=1-\Omega_{\Lambda}\right.$ here), and the normalization massdensity parameter $\sigma_{8}$.

Note that the linear analysis of the velocity data addresses the scaled power spectrum $P(k) \Omega_{\mathrm{m}}^{1.2}$ rather than $P(k)$ itself, and it therefore constrains the combination of parameters $\sigma_{8} \Omega_{\mathrm{m}}^{0.6}$, which serves as a measure of the power-spectrum amplitude. This result is almost independent of $\Omega_{\Lambda}$ (Lahav et al. 1991). Its shape is controlled by another combination $\Gamma \sim \Omega_{\mathrm{m}} h$. These combinations are therefore the natural parameters for the velocity analysis. The wavenumber range covered is roughly $0.05<k\left(h \mathrm{Mpc}^{-1}\right)<0.2$.

In order to account for non-linear effects acting on small scales, we add to the linear velocity correlation model an additional free parameter, $\sigma_{\mathrm{v}}$, representing an uncorrelated velocity dispersion at zero lag. This is a simple way to model small-scale random virial motions, but it can also be interpreted as an addition to the errors that enter the likelihood analysis. The parameter $\sigma_{\mathrm{v}}$ is allowed to vary together with the other model parameters. By this procedure, the cosmological parameters of interest are properly determined by the linear part of the fluctuations on large scales, while the undesired non-linear effects are detached and 'absorbed' by the additional free parameter. This procedure has been explored already in Freudling et al. (1999; Section 6.3.2), who obtained for the SFI catalogue a best-fitting value of $\sigma_{\mathrm{v}}=200 \pm 120 \mathrm{~km} \mathrm{~s}^{-1}$, resulting in values of $\sigma_{8} \Omega_{\mathrm{m}}^{0.6}$ lower by $10-20$ per cent than the values obtained without this additional term. This and other ways of correcting for non-linear effects have been found recently to provide consistent and more reliable results, based on improved mock catalogues drawn from high-resolution simulations and a principal-component analysis (Silberman et al., in preparation). However, it is not clear what value this additional free parameter should take. Since we consider it to crudely account for all possible non-linear effects, not necessarily restricted to the intrinsic velocity dispersion of galaxies, we may expect its value to be different from local estimates of velocity dispersion (e.g. $\sim 100 \mathrm{~km} \mathrm{~s}^{-1}$, van den Bergh 1999). For example, Riess et al. (1997) assume a redshift error of $200 \mathrm{~km} \mathrm{~s}^{-1}$ as an estimate of the contribution from non-linear effects. In the absence of any other information, we have carried out the Bayesian procedure for the case where we have no knowledge of a free parameter: we have marginalized over $\sigma_{\mathrm{v}}$. By marginalization we mean integrating likelihoods over a fixed range with a uniform prior. Since there is a weak constraint on $\sigma_{\mathrm{v}}$ from the peculiar velocity data themselves (e.g. Freudling et al. 1999), a range of integration from $0 \mathrm{~km} \mathrm{~s}^{-1}$ to $400 \mathrm{~km} \mathrm{~s}^{-1}$ is sufficiently large that the exact values of the limits make no difference to the results. However, the integration over $\sigma_{\mathrm{v}}$ inevitably widens the error bars (or error surface, see Fig. 3 in Section 3) from peculiar velocities, therefore we also comment on the level of agreement between data sets where $\sigma_{\mathrm{v}}$ is fixed at values of 0 and $200 \mathrm{~km} \mathrm{~s}^{-1}$.

Fig. 1(a) shows the two-dimensional probability distribution for the PV data alone in the parameter space $\left(\sigma_{8} \Omega_{\mathrm{m}}^{0.6}, \Omega_{\mathrm{m}}(h)\right.$ (the constraints in the $\sigma_{8} \Omega_{\mathrm{m}}^{0.6}, \Omega_{\mathrm{m}} h$ plane are virtually insensitive to the value of $\Omega_{\mathrm{m}}$, but for definiteness we have marginalized over $\Omega_{\mathrm{m}}$ ). The velocity data constraints at the 95 per cent confidence level are $0.48<\sigma_{8} \Omega_{\mathrm{m}}^{0.6}<0.86$ and $0.16<\Omega_{\mathrm{m}} h<0.58$, with roughly uncorrelated errors. For comparison, without the $\sigma_{\mathrm{v}}$ term the results are $0.65<\sigma_{8} \Omega_{\mathrm{m}}^{0.6}<0.89$ and $0.25<\Omega_{\mathrm{m}} h<0.66$. 


\subsection{The cosmic microwave background}

We use the same compilation of CMB anisotropy measurements as in Bridle et al. (1999), supplemented by the new TOCO points (Miller et al. 1999), the BOOMERANG North American test flight results (Mauskopf et al. 1999), the BOOMERANG 10-d Antarctica flight (de Bernardis et al. 2000) and the MAXIMA-1 results (Hanany et al. 2000). Since window functions are not yet available for these last three experiments we assume, for each band power estimate, Gaussian window functions that fall by a factor of $1 / \mathrm{e}$ at $\ell_{\min }$ and $\ell_{\max }$ as specified in de Bernardis et al. (2000) and Hanany et al. (2000). We also marginalize over the 10 and 4 per cent calibration uncertainties quoted, respectively, for
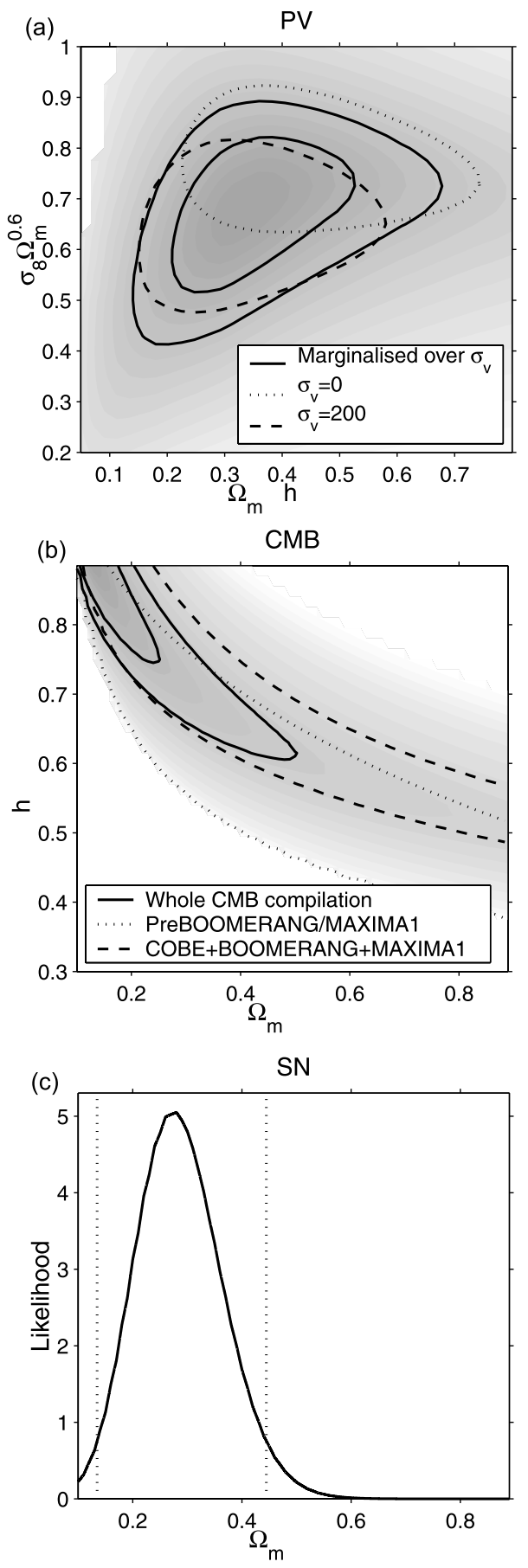

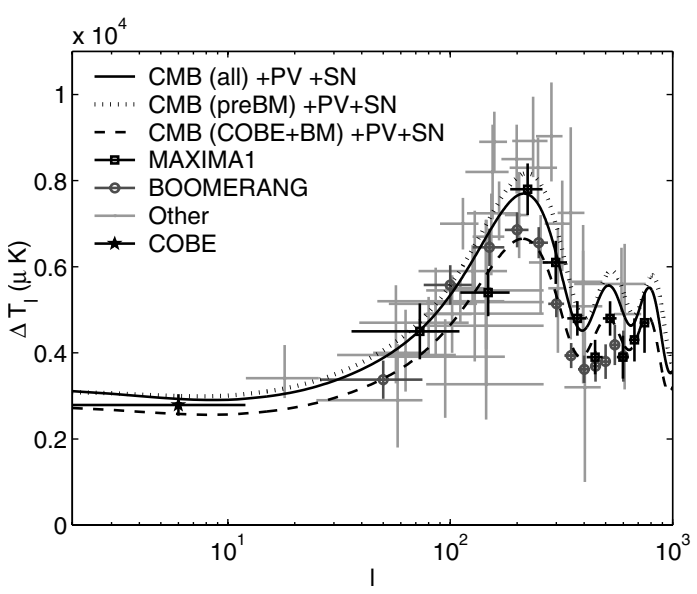

Figure 2. The CMB data used. The full curve is the theoretical power spectrum for the best-fitting point to the PV, SN and CMB when the whole CMB data compilation is used $\left(h=0.74, \Omega_{\mathrm{m}}=0.28, \sigma_{8}=1.16\right)$. The dotted curve is the best fit to $\mathrm{PV}+\mathrm{CMB}+\mathrm{SN}$ when just the preBOOMERANG (Antarctica flight)/MAXIMA-1 CMB data (pre-BM) is used ( $\left.h=0.65, \Omega_{\mathrm{m}}=0.34, \sigma_{8}=1.09\right)$. The broken curve is from when just $C O B E$, BOOMERANG (Antarctica flight) and MAXIMA-1 are used in the CMB compilation $\left(h=0.75, \Omega_{\mathrm{m}}=0.29, \sigma_{8}=1.09\right)$.

the BOOMERANG Antarctica and MAXIMA-1 results, fully taking into account the correlated nature of the calibration errors (Bridle et al., in preparation). The full compilation is plotted in Fig. 2. We compute the likelihood of the angular power spectra using the flat-band power method (e.g. Hancock et al. 1998). In addition to the assumptions already listed in the previous section, we assume there is negligible re-ionization and that there are negligible tensor contributions, as predicted by most inflation models. We obtain theoretical CMB power spectra as a function of the cosmological parameters using the CMBFAST and CAMB codes (Seljak \& Zaldarriaga 1996; Lewis, Challinor \& Lasenby 2000). In order to relate $\sigma_{8}$ to the CMB power spectrum normalization, we first relate $\sigma_{8}$ to the primordial matter power spectrum amplitude and then use the analytic expression from Efstathiou, Bond \& White (1992) to relate this to the $\ell=2$ amplitude of the CMB power spectrum.

The COBE data constrain the large-scale temperature fluctuations well, which converts to a strong constraint on $\sigma_{8}$ for given values of $h$ and $\Omega_{\mathrm{m}}$. The CMB data indicate the position of the first acoustic peak, near $\ell \sim 200$ which corresponds to a wavenumber of $k \sim 0.03 \mathrm{~h} \mathrm{Mpc}^{-1}$. This constrains the combination $\Omega_{\mathrm{m}}+\Omega_{\Lambda}$ to be roughly around unity (e.g. Efstathiou et al. 1999;

Figure 1. (a) The constraints from the peculiar velocity data plotted in the $\Omega_{\mathrm{m}} h, \sigma_{8} \Omega_{\mathrm{m}}^{0.6}$ plane (marginalized over $\Omega_{\mathrm{m}}$ ). The full curves show 68 and 95 per cent confidence limits after marginalization over $\sigma_{\mathrm{v}}$, which is used for the main part of the analysis. The dotted curve shows the 95 per cent constraint if the parameter $\sigma_{\mathrm{v}}$ is not used (or equivalently, $\sigma_{\mathrm{v}}=0$ ) the effect of which on the results of the joint analysis is discussed in Section 3. The result of setting $\sigma_{\mathrm{v}}=200 \mathrm{~km} \mathrm{~s}^{-1}$, the best-fitting value to $\mathrm{PV}$, is also shown (broken curve is the 95 per cent contour). (b) The constraints in the $\Omega_{\mathrm{m}}-h$ plane from the CMB data (marginalized over $\sigma_{8}$ ). The full curves show the 68 and 95 per cent limits using the whole CMB data compilation, which is used for that main results of this paper. The 95 per cent contours from the pre-BOOMERANG (Antarctica flight)/MAXIMA-1 data (pre$\mathrm{BM}$ ) and from just COBE + BOOMERANG (Antarctica flight)+ MAXIMA-1 data are shown by the dotted and broken curves, respectively. (c) The supernova constraint on $\Omega_{\mathrm{m}}$; the dotted curve shows the 95 per cent confidence limits. 

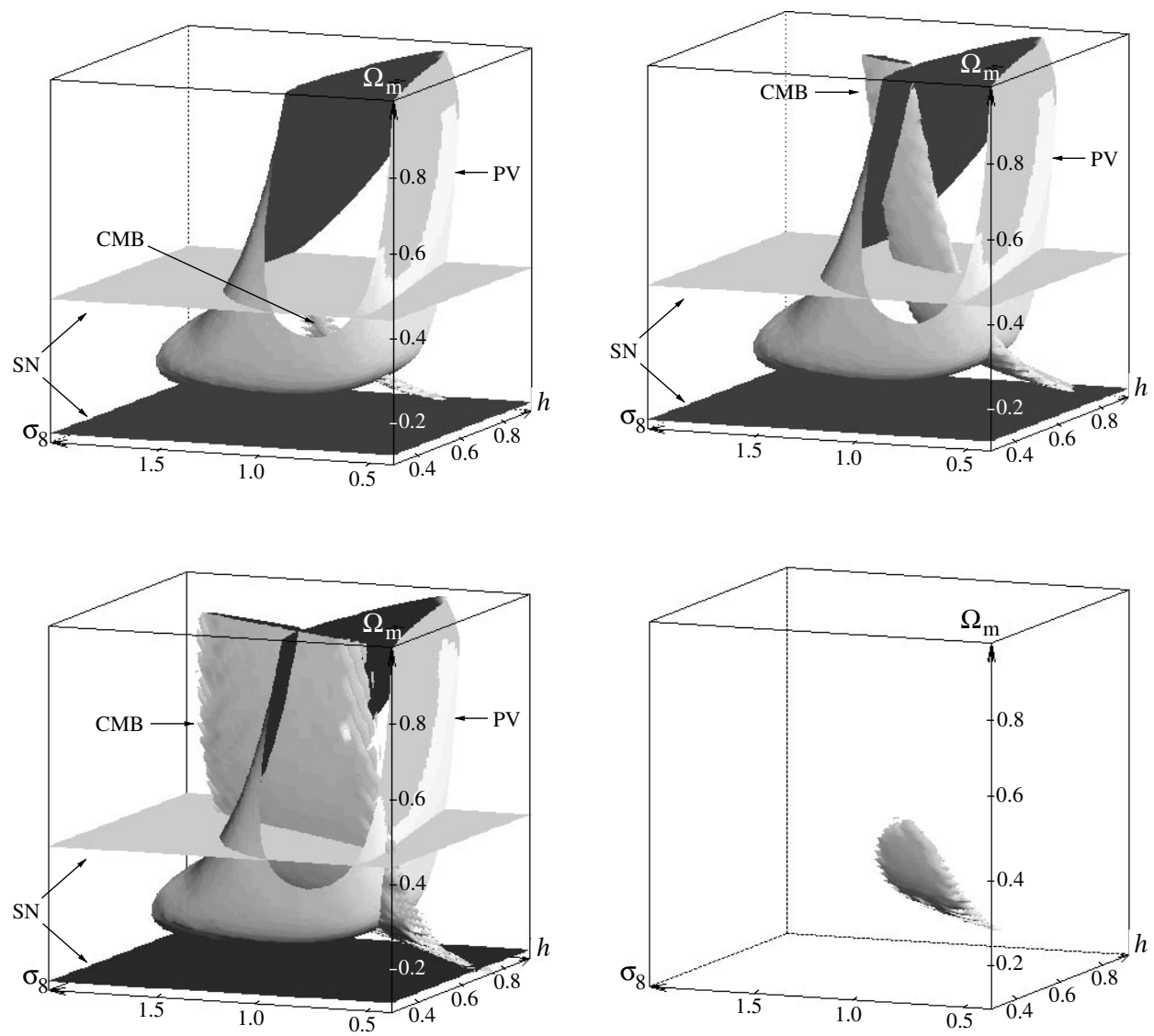

Figure 3. Top left: PV, CMB (whole compilation) and SN $2 \sigma$ iso-probability surfaces. For PV and CMB the surfaces are at $\Delta \log (\mathrm{Likelihood})=4.01$, and for the SN the surfaces are at $\Delta \log ($ Likelihood $)=2.00$, corresponding to the 95 per cent limits for three- and one-dimensional Gaussian distributions, respectively. (Integration under the likelihood surfaces can also be used instead of the likelihood itself in order to define 95 per cent limits, but this causes little difference to the allowed regions, in the cases shown here.) The SN surfaces are two horizontal planes. Top right: the same, but this time the data used for the CMB surface is just COBE, BOOMERANG (Antarctica flight) and MAXIMA-1. Bottom left: the same but this time the data used for the CMB surface is the pre-BM data. Bottom right: the 2- $\sigma$ surface for the joint PV, CMB (whole compilation) and SN likelihood function.

Balbi et al. 2000; Dodelson \& Knox 2000; Lange et al. 2000; Tegmark \& Zaldarriaga 2000), consistent with the flat universe assumed in our current analysis. In fact, using just BOOMERANG and $C O B E$, Lange et al. (2000) find $\Omega_{\mathrm{m}}+\Omega_{\Lambda} \sim 1.1$ (Fig. 2), whereas using just MAXIMA-1 and COBE, Balbi et al. (2000) find $\Omega_{\mathrm{m}}+\Omega_{\Lambda} \sim 0.9$. At $\sim 1^{\circ}$ angular scales the height of the first acoustic peak constrains the matter-radiation ratio at last scattering, which is proportional to $\Omega_{\mathrm{m}}(h)^{2}$. In addition, given our assumption of a flat universe, $\Omega_{\mathrm{m}}$ and $h$ also significantly affect the position of the first acoustic peak [see fig. 2 of White, Scott \& Pierpaoli (2000) for an illustration]. Increasing $\Omega_{\mathrm{m}}$ moves the peak to lower $\ell$, as does increasing $h$. These two effects combine to give the likelihood distribution in the $\Omega_{\mathrm{m}}-h$ plane shown in Fig. 1(b). The slightly lower first peak height indicated by the BOOMERANG and MAXIMA-1 data and the lower $\ell$ position of the first peak from the BOOMERANG data produce a constraint at higher $\Omega_{\mathrm{m}}$ and $h$ than does the pre-BOOMERANG/MAXIMA-1 compilation (hereafter pre-BM). Using the whole compilation together defines a region in $\left(\Omega_{\mathrm{m}}, h\right)$ space at the intersection of the BOOMERANG+ MAXIMA-1 and the pre-BM contours. This occurs at high $h$ and low $\Omega_{\mathrm{m}}$. It is interesting to note that the degeneracy directions for each of the pre-BM and the BOOMERANG+ MAXIMA-1 data sets are somewhat different (as shown in Fig. 1b). One possible explanation for this is that the older data put a strong constraint on the peak height, which is a function of $\Omega_{\mathrm{m}}(h)^{2}$. On the other hand the BOOMERANG + MAXIMA-1 data, with their detailed $\ell$ space coverage but significant calibration uncertainties, place a strong constraint on the peak position. Lines of constant peak position lie more parallel to the $\Omega_{\mathrm{m}}$ axis than do lines of constant $\Omega_{\mathrm{m}} h^{2}$ [as derived from Efstathiou \& Bond (1999) in Bridle, in preparation]. Therefore, using the whole CMB compilation allows tighter constraints to be placed on $h$ and $\Omega_{\mathrm{m}}$.

\subsection{Type Ia supernovae}

We use the constraints obtained by Perlmutter et al. (1999), which are fully consistent with those of Riess et al. (1998), based on applying the classical luminosity-redshift test to distant type Ia supernovae. The sample consists of 42 high-redshift SN $(0.18 \leqslant$ $z \leqslant 0.83)$, supplemented by 18 low-redshift $\mathrm{SNe}(z<0.1)$. This analysis determines a combination of $\Omega_{\mathrm{m}}$ and $\Omega_{\Lambda}$. Note that, unlike PV and CMB, SN are insensitive to the form of the matter power spectrum and depend only on the overall geometry of the universe. Since we limit ourselves in this paper to a flat universe, the SN constraint is translated to a likelihood function of $\Omega_{\mathrm{m}}$, shown in Fig. 1(c).

\section{COMPARISON AND COMBINATION}

In order to examine how well the constraints from PV, CMB and 
$\mathrm{SN}$ agree with each other we plot in Fig. 3 the three corresponding iso-likelihood surfaces, at the 2- $\sigma$ level, in the three-dimensional parameter space $\left(h, \sigma_{8}, \Omega_{\mathrm{m}}\right)$.

The upper and lower 95 per cent limits on $\Omega_{\mathrm{m}}$ from SN are the two horizontal planes. The PV surface encloses a space at roughly constant $\Omega_{\mathrm{m}}(h)$ and $\sigma_{8} \Omega_{\mathrm{m}}^{0.6}$. The CMB surface lies in the intersection of the regions allowed by each of SN and PV. The fact that the constraints have a common region of overlap is not trivial; it indicates a reasonable goodness of fit between the three data sets within the framework of the assumed cosmological model, which justifies a joint likelihood analysis aimed at parameter estimation. To illustrate the complementary nature of these three data sets we show in the top right-hand panel of Fig. 3 the same surfaces as in the top left-hand panel except that this time the CMB surface was calculated using only the $C O B E$, BOOMERANG (Antarctica flight) and MAXIMA-1 data. The CMB surface can be seen to be roughly orthogonal to each of the $\mathrm{PV}$ and SN surfaces. Also for comparison the result of using the pre-BM CMB data instead is shown in the bottom left-hand panel of Fig. 3. We have also looked at the equivalent to Fig. 3(a) where the peculiar velocity surface has $\sigma_{\mathrm{v}}$ fixed at $200 \mathrm{~km} \mathrm{~s}^{-1}$ instead of having been marginalized over. As expected, the peculiar velocity surface is smaller, but even so there is still a significant region of parameter space enclosed by all three surfaces.

Given the very different nature of the three data sets and the different redshift ranges probed by them $(z \sim 0.02,0.5,1000$ for $\mathrm{PV}, \mathrm{SN}$ and $\mathrm{CMB}$, respectively), we assume that the errors on the individual data sets are uncorrelated with each other. The likelihood of a given set of cosmological parameters is thus obtained by multiplying the three likelihoods of the parameters derived for each data set alone. The 2- $\sigma$ iso-probability surface for the joint likelihood function is shown in the bottom right-hand panel of Fig. 3. As expected, it is located at the intersection of the surfaces from each of the three data sets alone.

The best-fitting cosmological parameters $\left(\Omega_{\mathrm{m}}, h, \sigma_{8}\right)$ given all three data sets are given in Table 1 , from which we can derive $\sigma_{8} \Omega_{\mathrm{m}}^{0.6}=0.54, \Omega_{\mathrm{m}}(h)=0.21, Q_{\mathrm{rms}-\mathrm{ps}}=19.7 \mu \mathrm{K}$ and the age of

Table 1. Parameter values at the joint PV, CMB, SN optimum. The 95 per cent confidence limits are given, calculated for each parameter by marginalizing the likelihood function over the other parameters.

\begin{tabular}{lcc}
\hline Parameter & Best-fitting point & 95 per cent confidence limits \\
\hline$h$ & 0.74 & $0.64<h<0.86$ \\
$\Omega_{\mathrm{m}}$ & 0.28 & $0.17<\Omega_{\mathrm{m}}<0.39$ \\
$\sigma_{8}$ & 1.17 & $0.98<\sigma_{8}<1.37$ \\
\hline
\end{tabular}

the universe is $13.2 \mathrm{Gyr}$. The CMB power spectrum for this set of parameters is the full curve plotted in Fig. 2, which can be seen to be a reasonable fit to the data up to the end of the first acoustic peak. The $\chi^{2}$ with the CMB data is not simple to quote, since we have marginalized over the calibration uncertainties for the BOOMERANG and MAXIMA-1 data. However, using the bestfitting point to $\mathrm{CMB}$ (all data) $+\mathrm{PV}+\mathrm{SN}$, the $\chi^{2}$ with the pre$\mathrm{BM}$ data is 52. This is higher than the number of data points, 39, which reflects the fact that the BOOMERANG and MAXIMA-1 points are somewhat below the other CMB data points. Similarly for peculiar velocities, we have marginalized over $\sigma_{\mathrm{v}}$ before calculating the best-fitting point to $\mathrm{PV}+\mathrm{CMB}+\mathrm{SN}$, but for $\sigma_{\mathrm{v}}=200$ (the best-fitting value using peculiar velocities alone) the $\chi^{2}$ for the joint best-fitting point is 1155 , which is very similar to the number of data points, 1156.

We may evaluate the probability of a single cosmological parameter, independent of the values of the other cosmological parameters, by integrating the probability over the values of the other parameters. This is what we mean by 'marginalization'. The full curves in Fig. 4 shows the resulting one-dimensional marginalized likelihood distributions for each parameter. We obtain the 95 per cent limits by integrating the one-dimensional likelihood distributions and requiring that 95 per cent of the probability lies between the quoted limits. These limits are those presented in Table 1. The $h$ range agrees well with that from the Hubble Space Telescope (HST) key project of $h=0.71 \pm 0.06$ (Mould et al. 1999) and the $\Omega_{\mathrm{m}}$ limits are roughly centred on the popular value of 0.3 .

Fig. 5 shows together the two- and one-dimensional (1D) marginalized distributions as evaluated for each data set alone and then jointly for each pair of data sets, and finally for the three data sets together (again, the 68 and 95 per cent limits are found by integration of the probability distributions). For the pairs of data sets, or the single data sets alone, there is some dependence of the confidence regions on the ranges used in the marginalization. However, the results when all three data sets are used are insensitive to the ranges of integration we have used, except for the limit of $h<0.9$, which we consider to be a reasonable prior. A measure of the excellent agreement between these data sets is given by the similarity of the parameter constraints from the three different possible pairings of the data sets (1D plots marked $\mathrm{P}+\mathrm{S}$, $\mathrm{P}+\mathrm{M}$ and $\mathrm{S}+\mathrm{M}$ in Fig. 5). Also note that the $\mathrm{CMB}$ data alone prefer a high $h$, but on combining with $\mathrm{PV}$ and $\mathrm{SN}$ there is an upper bound that is just below our prior of $h<0.90$. A detailed examination in three dimensions reveals that inclusion of the PVs cuts off a high- $h$, low- $\Omega_{\mathrm{m}}$ part of the CMB surface, and inclusion of SN cuts off a high- $h$, high- $\Omega_{\mathrm{m}}$ part of the CMB surface, thus lowering the preferred value of $h$.
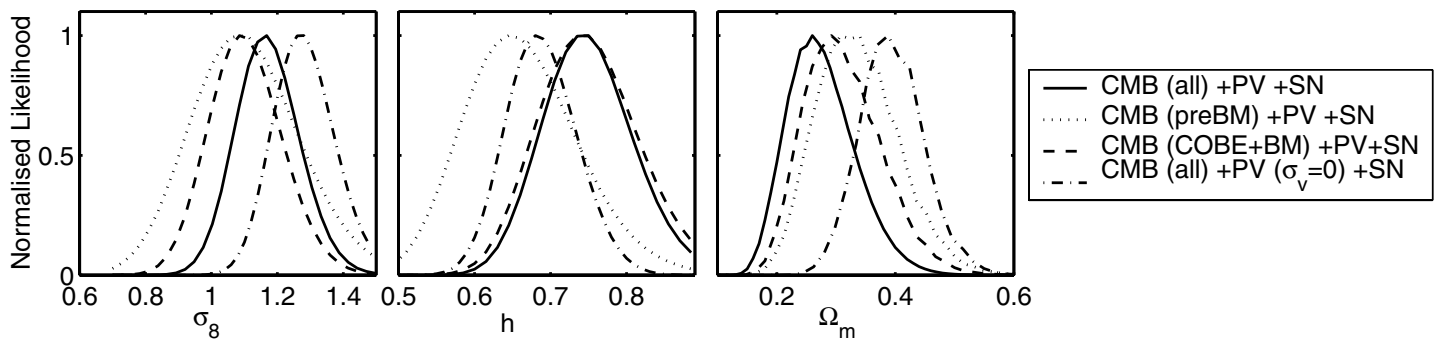

Figure 4. The one-dimensional marginalized likelihood distributions from the joint PV, CMB and SN likelihood function. Our main results are shown by the full curves, which use the whole CMB data compilation, PV (marginalized over $\sigma_{\mathrm{v}}$ ) and SN. The dotted curves show the likelihood functions when PV, SN and just the pre-BM CMB data are used. The broken curve is the result when the CMB data are just COBE + BOOMERANG + MAXIMA-1. The result (using all the CMB data) when the uncorrelated velocity dispersion term is not included in the PV analysis $\left(\sigma_{\mathrm{v}}=0\right)$ is shown by the chain curve. 

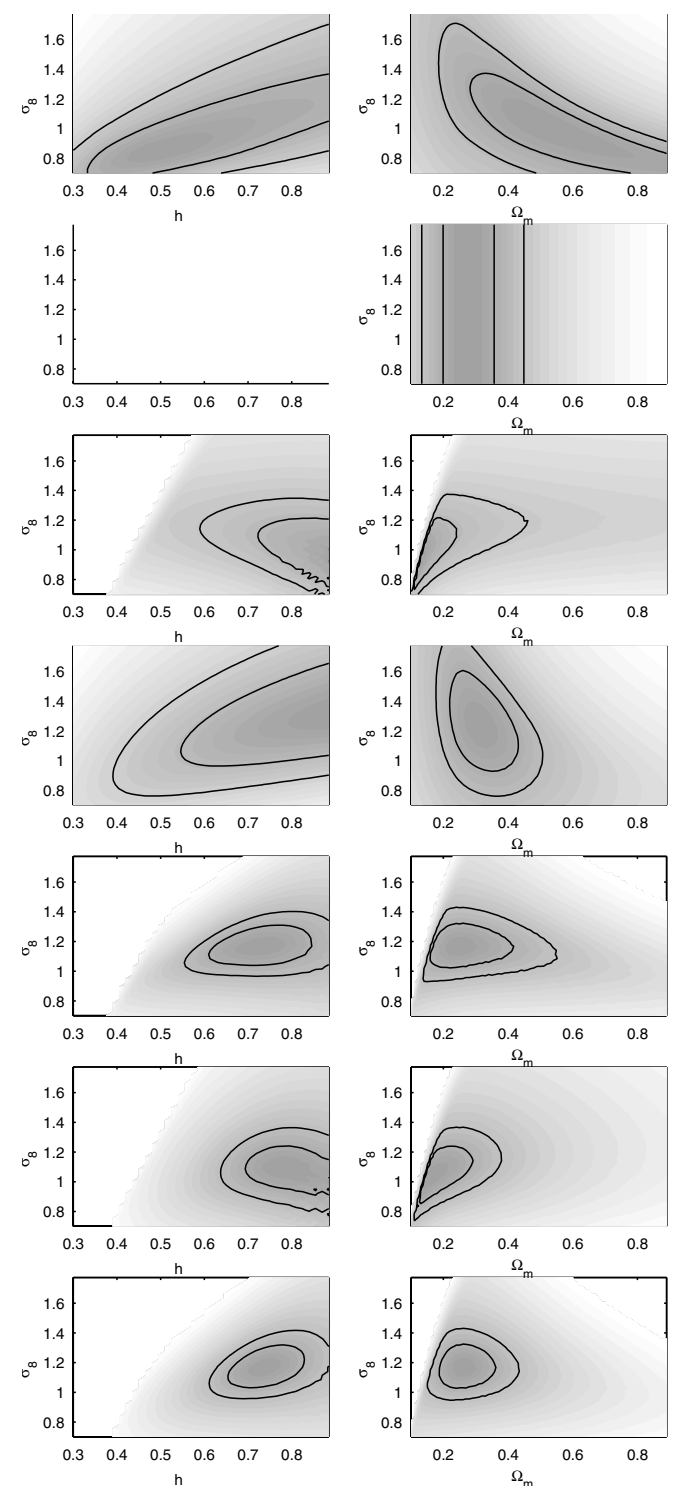
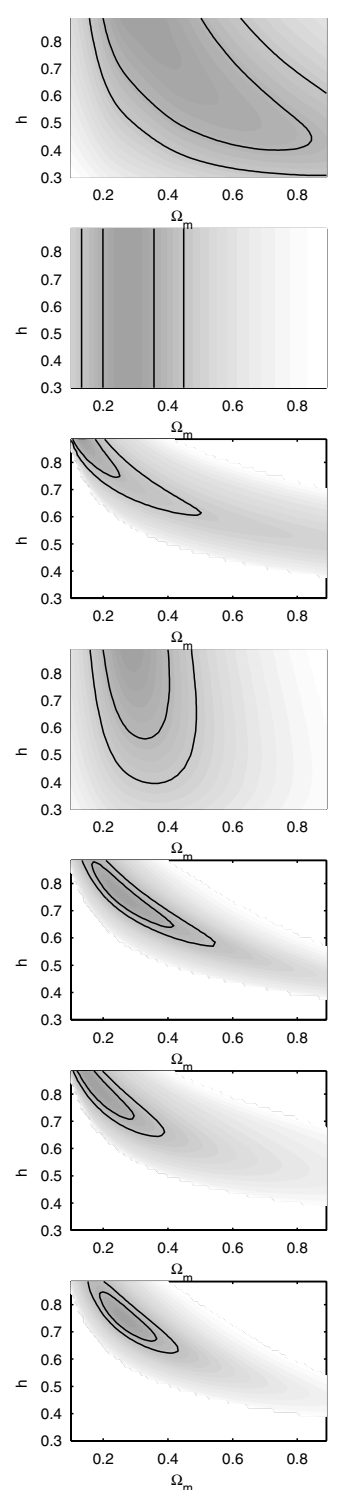
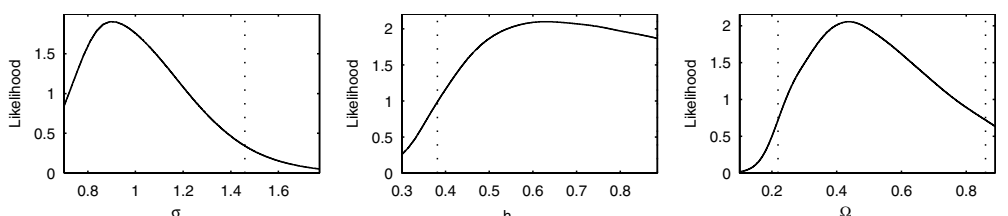

Pec Vels (P)
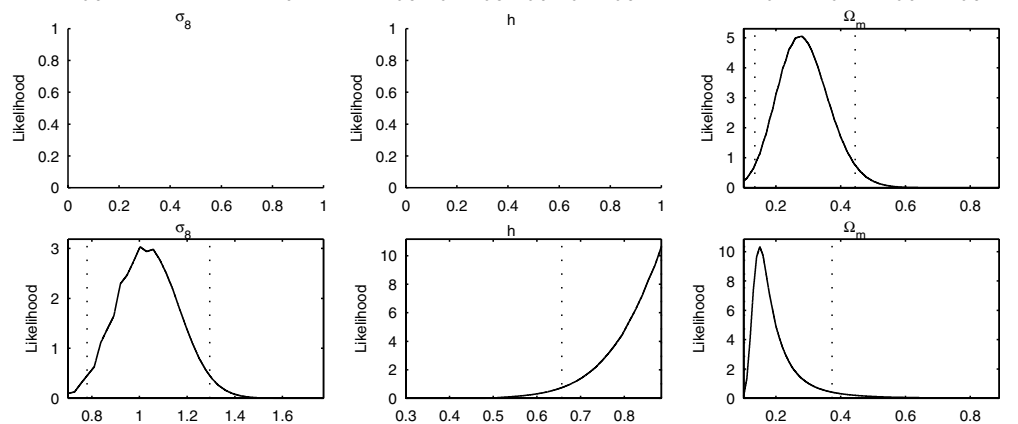

CMB (M)
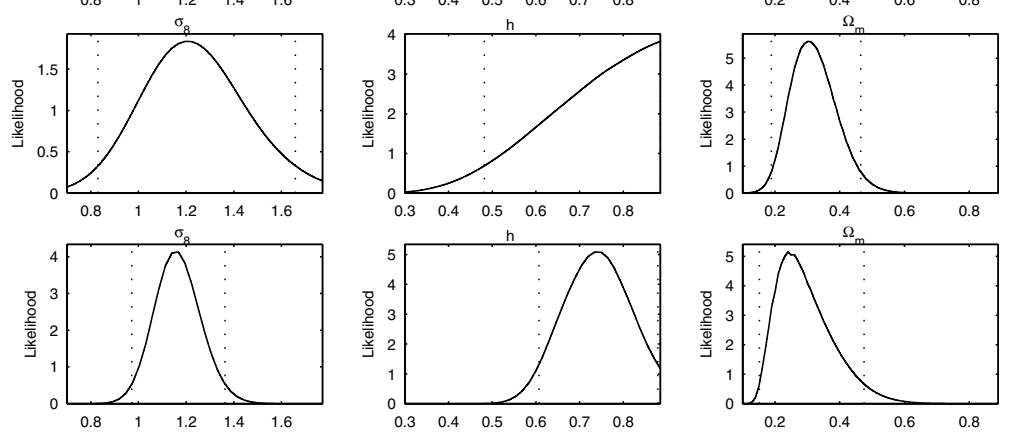

$\mathrm{P}+\mathrm{M}$
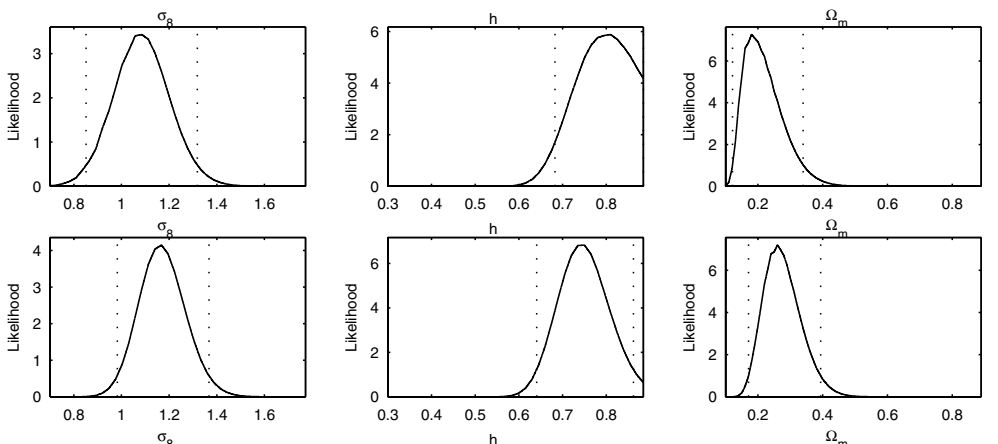

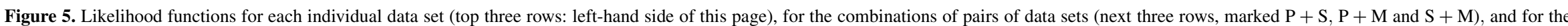

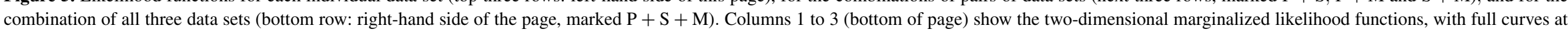

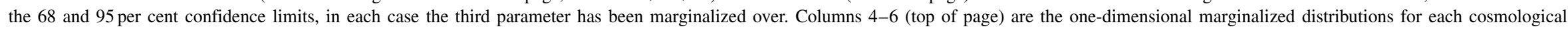
parameter, with dotted curves showing the 95 per cent confidence regions. Note that the whole CMB data compilation was included in this analysis, and the PV term $\sigma_{\mathrm{v}}$ was marginalized over 
We have repeated the entire analysis using different subsets of CMB data. Using the pre-BM data the one-dimensional marginalized likelihood functions (dotted curves in Fig. 4) are in good agreement but somewhat wider than when using all the data, especially in the constraint on $h$ which extends to lower values than before. Using just the COBE, BOOMERANG (Antarctica flight) and MAXIMA-1 data (broken curves) the results are very similar to when all data are used. At first this may seem surprising given the much larger three-dimensional surface [top right versus top left-hand panels of Fig. 3 and Fig. 1(b)] but the high $\Omega_{\mathrm{m}}, h$ part is ruled out by both PV and SN, leaving virtually the same region as when all CMB data are used.

In the region of the power spectrum where a second acoustic peak is predicted, we note that our best-fitting models are not a good fit to the data, producing more power than observed by both BOOMERANG and MAXIMA-1. The easiest way to reconcile this is to increase $\Omega_{\mathrm{b}}(h)^{2}$ to a value approximately double that found from nucleosynthesis (e.g. Hu 2000; White et al. 2000). This has the effect of increasing the heights of the odd numbered peaks and decreasing those of the even numbered peaks. It also has the effect of reducing the sound horizon and thus shifting the first peak to even smaller angular scales. Repeating some of our analysis using $\Omega_{\mathrm{b}} h^{2}=0.04$ (and assuming that the PV likelihood function is relatively insensitive to this value) we find that the agreement between the data sets is still good, and the constraints on $\sigma_{8}$ and $\Omega_{\mathrm{m}}$ are not significantly affected. However, the bestfitting $h$ value tends towards our upper limit of 0.90 , which allows the peak to be at smaller angular scales (White et al. 2000; Fig. 2).

In order to check the level of sensitivity to the use of $\sigma_{\mathrm{v}}$ in the $\mathrm{PV}$ analysis we have also repeated the joint analysis using peculiar velocity likelihoods that were obtained without including this additional term (Section 2.1). This is the linear method used in the main section of Freudling et al. (1999) and in Zehavi \& Dekel (1999). As mentioned already, the linear analysis prefers slightly higher values of $\sigma_{8} \Omega_{\mathrm{m}}^{0.6}$. As a result, the region of agreement between PVs, CMB and SN now only just occurs at the 2- $\sigma$ level. The resulting 1D marginalized likelihood functions are the chain curves in Fig. 4. They are not very different from those obtained with the non-linear correction, having slightly higher $\Omega_{\mathrm{m}}$ and $\sigma_{8}$ and a slightly lower $h$. It is encouraging to see that the joint analysis results are fairly robust to the uncertainties in the PV analysis.

We also quote the results for our main analysis (whole CMB data compilation, marginalized over $\sigma_{\mathrm{v}}$ ) in terms of the natural parameter combinations for PVs in Table 2. The range in $\sigma_{8} \Omega_{\mathrm{m}}^{0.6}$ is slightly lower than that preferred by peculiar velocities alone, mainly owing to the orthogonal constraint from the CMB on $\sigma_{8}$ and $\Omega_{\mathrm{m}}$ in the $\Omega_{\mathrm{m}}$ range allowed by SN, which disfavours larger $\sigma_{8} \Omega_{\mathrm{m}}^{0.6}$. The $\Omega_{\mathrm{m}}(h)$ limits are much tighter and at the low end of those provided by peculiar velocities alone, again mainly owing to the CMB constraint in the range allowed by $\mathrm{SN}$.

Table 2. Parameter values at the joint PV, CMB, SN optimum and 95 per cent limits in terms of the parameters $\sigma_{8} \Omega_{\mathrm{m}}^{0.6}, \Omega_{\mathrm{m}} h$ and $\Omega_{\mathrm{m}}$.

\begin{tabular}{lcc}
\hline Parameter & Best-fitting point & 95 per cent confidence limits \\
\hline$\sigma_{8} \Omega_{\mathrm{m}}^{0.6}$ & 0.54 & $0.40<\sigma_{8} \Omega_{\mathrm{m}}^{0.6}<0.73$ \\
$\Omega_{\mathrm{m}} h$ & 0.23 & $0.16<\Omega_{\mathrm{m}} h<0.27$ \\
$\Omega_{\mathrm{m}}$ & 0.28 & $0.18<\Omega_{\mathrm{m}}<0.42$ \\
\hline
\end{tabular}

\section{CONCLUSION}

We have performed a joint analysis of three complementary data sets free of galaxy-density biasing, using peculiar velocities, CMB anisotropies and high-redshift supernovae. The constraints from the three data sets overlap well at the $2-\sigma$ level and there is an acceptable goodness of fit. These data sets constrain roughly orthogonal combinations of the cosmological parameters, and are combined to provide tighter constraints on the parameters (Table 1). These constraints are found to be fairly robust to the CMB data compilation used, the peculiar velocity catalogue used, and the assumption of an uncorrelated velocity dispersion at zero lag (Fig. 4).

The values obtained from the joint analysis for $h$ and $\Omega_{\mathrm{m}}$, and for the combinations of cosmological parameters (Table 2), are in general agreement with other estimates (e.g. Bahcall et al. 1999), but this analysis tends to favour a slightly higher value for $\sigma_{8}$. In particular, the result for $\sigma_{8}$ is higher than the constraint of Bridle et al. (1999), $\sigma_{8}=0.74 \pm 0.1$ (95 per cent confidence) obtained by combining the $\mathrm{CMB}$ with cluster abundance and IRAS and allowing for linear biasing. This may reflect the preference of the peculiar velocities for a slightly higher value of $\sigma_{8} \Omega_{\mathrm{m}}^{0.6}$ than favoured by the cluster abundance analysis. The implications of considering the constraints arising from all the above-mentioned probes will be discussed elsewhere.

A recent linear analysis of a new peculiar velocity survey of early-type galaxies (ENEAR) by Zaroubi et al. (2000) finds similar results to Mark III and SFI, with a somewhat higher amplitude for the power spectrum and the indicated value of $\Omega_{\mathrm{m}}$. This is indeed the kind of non-linear bias expected in a sample of early-type galaxies that tend to be more clustered than the latetype galaxies dominating Mark III and SFI. With a non-linear correction of the sort employed in this paper, we can expect the results from ENEAR to become consistent with the constraints from Mark III and SFI (work in progress).

The addition of BOOMERANG and MAXIMA-1 to our CMB data compilation brought down the height of the first acoustic peak and shifted it to larger angular scales, which both increase a combination of $h$ and $\Omega_{\mathrm{m}}$. The combination of BOOMERANG and MAXIMA-1 with the older CMB data had the effect of breaking the degeneracy between $h$ and $\Omega_{\mathrm{m}}$ and leaving a high- $h$ region of parameter space. The resulting constraint on the Hubble constant, $h=0.75 \pm 0.11$ (95 per cent confidence), agrees well with that from the HST key project value of $h=0.71 \pm 0.06$. This result is also similar to that of Lange et al. (2000; table 1, p. 10).

Note that in this analysis we take all the data sets used at equal weight. An extension to this work would be to allow freedom in the weights given to the different probes, as in Lahav et al. (2000).

\section{ACKNOWLEDGMENTS}

We thank Graça Rocha for her work on compilation of the CMB data set and George Efstathiou for providing the supernova likelihoods. We thank the referee, Saleem Zaroubi for helpful comments. We also acknowledge useful discussions with Carlos Frenk on the role of cluster abundances. SLB acknowledges the PPARC for support in the form of a research studentship. This work was supported by the DOE and the NASA grant NAG 5-7092 at Fermilab, by US-Israel Binational Science Foundation grants 95-00330 and 98-00217, and by Israel Science Foundation grant 546/98. SLB and IZ acknowledge the hospitality of the Hebrew University of Jerusalem. 


\section{RE F E REN CES}

Bahcall N., Ostriker J., Perlmutter S., Steinhardt P., 1999, Science, 284, 1481

Balbi et al., 2000, ApJL, submitted (astro-ph/0005124)

Bond J. R., Jaffe A. H., 1998, Phil. Trans. R. Soc. Lond. A, Discussion Meeting on Large Scale Structure in the Universe. Royal Society, London, March 1998

Bridle S. L., Eke V. R., Lahav O., Lasenby A. N., Hobson M. P., Cole S., Frenk C. F., Henry J. P., 1999, MNRAS, 310, 565

Burles S., Nollett K. M., Truran J. N., Turner M. S., 1999, Phys. Rev. Lett., 82,4176

de Bernardis P. et al., 2000, Nat, 404, 955

Dodelson S., Knox L., 2000, Phys. Rev. Lett., 84, 3523

Efstathiou G., Bridle S. L., Lasenby A. N., Hobson M. P., Ellis R. S., 1999, MNRAS, 303, L47

Efstathiou G., Bond J. R., 1999, MNRAS, 304, 75

Efstathiou G., Bond J. R., White S. D. M., 1992, MNRAS, 258, 1P

Eke V. R., Cole S., Frenk C. S., Henry J. P., 1998, MNRAS, 298, 1145

Fisher K. B., Scharf C. A., Lahav O., 1994, MNRAS, 266, 219

Freudling W. et al., 1999, ApJ, 523, 1

Gawiser E., Silk J., 1998, Science, 280, 1405

Hanany S., et al., 2000, ApJL, submitted (astro-ph/0005123)

Hancock S., Rocha G., Lasenby A. N., Gutierrez C. M., 1998, MNRAS, 294, L1

Haynes M. P., Giovanelli R., Salzer J. J., Wegner G., Freudling W., da Costa L. N., Herter T., Vogt N. P., 1999a, AJ, 117, 1668

Haynes M. P., Giovanelli R., Chamaraux P., da Costa L. N., Freudling W., Salzer J. J., Wegner G., 1999b, AJ, 117, 2039

Hu W., Sugiyama N., Silk J., 1997, Nat, 386, 6

Hu W., 2000, Nat, 404, 939
Lahav O., Bridle S. L., Hobson M. P., Lasenby A. N., Sodre L., Jr, 2000, MNRAS, 315, L45

Lahav O., Lilje P. B., Primack J. R., Rees M. J., 1991, MNRAS, 251, 128

Lange A. E. et al., 2000, preprint (astro-ph/0005004)

Lewis A., Challinor A., Lasenby A., 2000, ApJ, 538, 473

Lineweaver C. H., 1998, ApJ, 505, L69

Mauskopf P. et al., 2000, ApJ, 536, L59

Miller A. et al., 1999, ApJ, 524, L1

Mould J. et al., 2000, ApJ, 529, 786

Perlmutter S. et al., 1999, ApJ, 517, 565

Riess A., Davis M., Baker J., Kirshner R., 1997, ApJ, 48, L1

Riess A. G. et al., 1998, AJ, 116, 1009

Seljak U., Zaldarriaga M., 1996, ApJ, 469, 437

Sugiyama N., 1995, ApJs, 100, 281

Tegmark M., Zaldarriaga M., 2000, preprint (astro-ph/0004393)

Tytler D., O'Meara J., Suzuki N., Lubin D., 2000, to appear in Physica Scripta (astro-ph/0001318)

van den Bergh S., 1999, A\&AR, 9, 273

White M., Scott D., Pierpaoli E., 2000, ApJL, submitted (astro-ph/ 0004385)

Willick J. A., Courteau S., Faber S. M., Burstein D., Dekel A., Strauss M. A., 1997, ApJS, 109, 333

Zaroubi S., Zehavi I., Dekel A., Hoffman Y., Kolatt T., 1997, ApJ, 486

Zaroubi S., Bernardi M., da Costa L., Hoffman Y., Alonso V. G., Willmer C., Pellegrini P., 2000, preprint (astro-ph/0005558)

Zehavi I., Dekel A., 1999, Nat, 401, 252

Zehavi I., Dekel A., 2000, in Courteau S., Strauss M. A., Willick J. A., eds, Proc. of Cosmic Flows 1999: Towards an Understanding of LargeScale Structure, ASP Conf. Series, Vol. 201

This paper has been typeset from a $\mathrm{T}_{\mathrm{E}} \mathrm{X} / \mathrm{L} \mathrm{T} \mathrm{E} \mathrm{X}$ file prepared by the author. 